\title{
Attention, Researchers! It Is Time to Take a Look at the Real World
}

\author{
Alan Kingstone, ${ }^{1}$ Daniel Smilek, Jelena Ristic, \\ Chris Kelland Friesen, and John D. Eastwood
}

\begin{abstract}
Department of Psychology, University of British Columbia, Vancouver, British Columbia, Canada (A.K.; D.S.; J.R.); Department of Psychology, North Dakota State University, Fargo, North Dakota (C.K.F.); and Department of Psychology, York University, Toronto, Ontario, Canada (J.D.E.)
\end{abstract}

\begin{abstract}
Theories of attention, too often generated from artificial laboratory experiments, may have limited validity when attention in the natural world is considered. For instance, for more than two decades, conceptualizations of "reflexive" and "volitional" shifts of spatial attention have been grounded in methodologies that do not recognize or utilize the basic fact that people routinely use the eyes of other people as rich and complex attentional cues. This fact was confirmed by our novel discovery that eyes will trigger a reflexive shift of attention even when they are presented centrally and are known to be spatially nonpredictive. This exploration of realworld attention also led to our finding that, contrary to popular wisdom, arrows, like eyes, are capable of producing reflexive shifts of attention-a discovery that brings into question much of the existing attention research. We argue that research needs to be grounded in the real world and not in experimental paradigms. It is time for cognitive psychology to reaffirm the difficult task of studying attention in a manner that has relevance to real-life situations.
\end{abstract}

\section{Keywords}

attention; eye gaze; situated cognition; ecological validity
The researcher remote from immediate practical pressures may indeed be free to study major variables in which at this instant society does not seem to be interested; but he should not use this freedom in order to study minor variables, until there are no major ones within reach of our techniques. The necessity for some relevance to real life is a worthwhile intellectual discipline. (Broadbent, 1971, p. 4)

Broadbent is one of the most significant researchers in experimental psychology. His research and writings on attention helped to initiate and fuel the paradigm shift known as the cognitive revolution. Broadbent was adamant that psychological theory must be grounded in real-life experiences. We argue, in part on the basis of recent findings from our lab, that cognitive psychology has, over time, lost touch with this foundation. The result is that theories of attention, often arising out of artificial laboratory experiments, fail to generalize to the real world. Thus, researchers are often unable to ask, let alone answer, many major questions regarding the everyday functioning of attention.

\section{THE CLASSIC ATTENTION PARADIGM}

In the late 1970s (Posner, 1978) and early 1980s (Jonides, 1981), a methodology that would come to be known as the Posner cuing paradigm was introduced. Since then, it has been used routinely to study human spatial attention. In this paradigm, a central fixation dot is flanked by two squares (boxes). The task is to make a key press as quickly as possible when a target item appears inside one of the boxes. This target event is preceded by a cue (the flashing of one of the boxes or the appearance of a central arrowhead pointing toward one of the boxes; see Figs. 1a and $1 b)$. The standard finding is that the target is detected faster when it appears in the cued box than when it appears in the uncued box. Because the brain processes attended items more quickly than unattended items, it is concluded that target detection time is speeded because attention is committed to the box that was cued.

Thus, there are two different ways that attention can be directed in the Posner paradigm. One way is to flash one of the boxes (Fig. 1a). In this case, attention is directed to the cued box that flashed. This attention shift is considered reflexive because people are faster to detect a target in the cued box even when the flashing does not predict where the target will occur (i.e., the target appears in the cued box $50 \%$ of the time and in the uncued box $50 \%$ of the time).

The other way to direct attention in this paradigm is to present a central arrowhead pointing left or right (Fig. 1b). In this case, attention is directed to the cued box that the arrowhead pointed toward. It has been assumed, however, that this happens only when the arrowhead predicts where the target will appear (e.g., the target appears in the cued box $80 \%$ of the time and in the uncued box $20 \%$ of the time), and not when the arrowhead is nonpredictive (Fig. 1c). Thus, the attentional shift associated with a central directional cue is considered voluntary. 


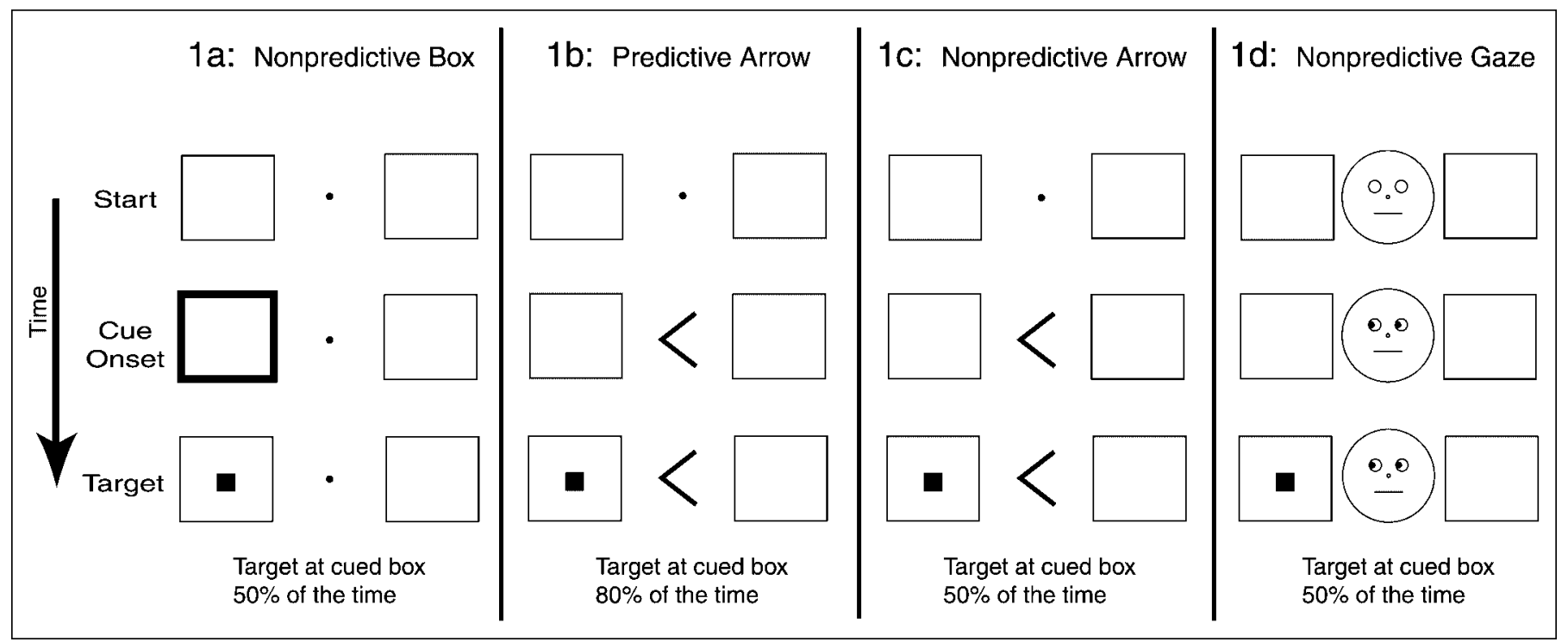

Fig. 1. Variations of the Posner paradigm. Each panel presents three stages of a typical trial (start, cue onset, and target onset); in these examples, the target (a small black square) appears at the cued box. In one variation of the paradigm (a), at the start of each trial, a central fixation dot is flanked by two squares (boxes). The left or right box is cued by a brief flash (illustrated by the thick black line), and then a target (the black square) is presented. The task is to press a key as quickly as possible when the target appears. The target appears in the cued (flashed) box 50\% of the time and in the uncued (not flashed) box 50\% of the time. Thus, the cue does not predict where the target will appear. In another variation (b), the left or right box is cued by an arrow pointing toward it, and the target appears in the cued box $80 \%$ of the time and in the uncued box $20 \%$ of the time. Thus, the cue predicts where the target will appear. In a third variation (c), the arrow cue is nonpredictive, as the target appears in the cued box $50 \%$ of the time and in the uncued box $50 \%$ of the time. In a fourth variation (d), the left or right box is cued by eyes looking toward it, and the target appears in the cued box $50 \%$ of the time and in the uncued box $50 \%$ of the time. Thus, the cue does not predict where the target will appear.

To what extent do these laboratory studies of simple attentional functions, such as detecting a light at cued or uncued boxes, have to do with the many experiences that people share every day, such as going to work, talking with friends, or eating a meal? On the face of it, not very much. Nevertheless, the assumption is that the principles of human attention and behavior studied in the laboratory do in fact have important implications for these everyday situations. In the words of Broadbent (1971, p. 3) “A man does not use one brain in the laboratory and another in the rest of his life." In other words, although there are many differences between the paradigms used in the laboratory and real-life situations, the assumption is that laboratory research exposes fundamental principles of human thought, attention, and behavior that will generalize to the everyday environment.

However, this assumption is not always valid. Indeed, our research suggests that laboratory studies conceived and interpreted in isolation from real-world experience may do far worse than fail to generalize back to the natural environment; they may generate fundamental misunderstandings of the principles of human attention. Specifically, our results demonstrate that, contrary to the accepted view, directional cues (such as arrows) that are centrally presented and spatially nonpredictive do lead to reflexive shifts of attention. It was our studies of attention as it might operate in the real world-namely, our studies of the role of eye gaze in directing attention-that ultimately revealed this fundamental error in the traditional view, as well as a basic error in scien- tists' understanding of how human attention is directed in the world.

\section{ATTENTION TO EYES}

Where other people look can reveal where they are attending, and thus indicate sources of potential interest in the environment. Gaze direction is such a powerful stimulus that the human eye, with its white background and darker iris, may have evolved to allow easy discrimination of small shifts in the eye direction of another individual (Kobayashi \& Kohshima, 1997). And the human brain may have evolved to be especially good at picking up these signals, with cells specialized for processing gaze information. Such processing is demonstrated at a very early age in 
healthy infants, with babies as young as 2 to 3 months old looking preferentially at eyes and discriminating changes in eye direction. Conversely, failures in gaze processing are linked to deficits in social cognition such as autism (BaronCohen, 1995).

We (Friesen \& Kingstone, 1998) hypothesized that because eyes are so important to human attention, shifts in eye direction might automatically trigger attention to gazedat locations. We tested this idea by modifying the Posner paradigm in two significant ways. First, arrows pointing to the left and right were replaced by a schematic face that looked left or right. Second, the predictive value of the central cue was eliminated; that is, eye direction did not predict where a target item would appear (see Fig. 1d). Note that because the eyes were centrally located and nonpredictive, the traditional line of thinking predicted that they would not lead to shifts of attention.

Remarkably, and contrary to the traditional way of thinking, eye gaze did trigger shifts of attention; target detection was faster for items at the gazed-at location than for items at the other location. This result led us to conclude that the attentional shift was reflexive because it emerged rapidly and in response to eyes that were spatially nonpredictive.

\section{OPENING ONE'S EYES TO THE WORLD}

Our experiments on eye gaze highlight the importance of studying attention with regard to the real world. What was taken as gospel-that a central directional stimulus must be spatially predictive to produce a shift in spatial attention-was found to be in error when tested with gaze cues.
This new perspective led us to reconsider the fundamental assumption underlying the role of arrows in directing attention. Since a classic study by Jonides (1981, Experiment 2), which failed to reject the null hypothesis that nonpredictive central arrows do not trigger orienting, researchers have assumed that arrows do not produce a shift in attention unless they predict where an item will appear. From the perspective of the real world, however, it would seem that arrows, like eyes, should produce automatic shifts in attention. This is because arrows are obviously very directional in nature, and, like eyes, they have a great deal of social significance. Indeed, it is a challenge to move through one's day without encountering any number of arrows on signs and postings.

We tested whether arrows produce reflexive shifts of attention by replacing spatially nonpredictive eyes with spatially nonpredictive arrows (Fig. 1c; Ristic, Friesen, \& Kingstone, 2002). The results were unequivocal. People attend to where arrows point even when they know that the arrows do not predict where a target will appear. In other words, like eyes, arrows produce a reflexive shift in attention to the cued location. The conclusion to be drawn from this finding is that any directional cue with social significance might produce a reflexive shift in spatial attention.

\section{WHAT HAVE ATTENTION RESEARCHERS BEEN MEASURING?}

If arrows produce reflexive shifts in attention when they are spatially nonpredictive, then what exactly have researchers been measuring for the past 20 years with predictive arrows? Were they measuring volitional attention, as they assumed? To answer this question, we (Ristic, Olk, Ho, \& Kingstone, 2003) compared pure measures of reflexive orienting and volitional orienting with a measure of orienting to predictive arrows. We measured reflexive orienting using spatially nonpredictive arrows and volitional orienting using predictive central cues that were not inherently directional. The attention effect with predictive arrows was greater than the effect with predictive nondirectional cues. The implication is that the effect found with a central predictive arrow cue does not reflect solely volitional orienting. Indeed, the effect with the predictive arrows was far greater than the sum of the measures of reflexive and volitional orienting, which suggests that this effect reflects some interaction that exceeds what is produced by two pure measures of volitional and reflexive attention, or perhaps some qualitatively unique form of attentional orienting.

\section{CONCLUSIONS AND FUTURE DIRECTIONS}

By adopting a real-world perspective, the research discussed here has demonstrated three major new findings, all contrary to what traditional thinking would have predicted: (a) Central nonpredictive eye gaze produces reflexive shifts of attention, (b) central nonpredictive arrows also produce automatic orienting, and (c) central predictive directional stimuli produce an attention effect that exceeds anything that could be expected by volitional orienting alone. Together, these findings raise several important practical and theoretical issues.

Perhaps the most fundamental issue raised by the research discussed here pertains to the validity of laboratory findings in real-world situations. Specifically, the evidence suggests that laboratory 
studies that have lost touch with real-life context may generate fundamental misunderstandings of the principles of human attention and behavior. It would be a mistake to think that our message applies only to the Posner paradigm. The same conclusion holds for many other laboratory paradigms used to study attention, such as the popular visual search paradigm, in which individuals look for a target hidden among a number of nontargets.

Studies of visual search have typically used very impoverished stimuli (e.g., colored rectangles) and have led to the development of several models in which attention is thought to be oriented only to primitive stimulus features (e.g., a unique color or shape) or some special combination of these primitive features (e.g., Treisman \& Gelade, 1980). In contrast, visual search studies that have used realworld stimuli have led to a much different conclusion. There is now a growing body of evidence showing that in visual search, attentional orienting can be triggered not only by primitive features but also by complex object properties like social significance. For instance, we have shown that attention can be oriented by the emotional expressions of faces (Eastwood, Smilek, \& Merikle, 2001). Thus, studies of visual search provide another illustration of how laboratory studies devoid of real-life context may generate fundamental misconceptions regarding the mechanisms underlying attention. Given this general problem, future research should focus on identifying which laboratory findings do, and do not, generalize to real-world situations. This seems especially relevant for studies that involve unnaturally controlled situations (e.g., neuroimaging investigations of brain activity investigations).

Another issue raised by the research we have discussed is that laboratory studies that are not mo- tivated or informed by real-life experience run the serious risk of excluding the exploration of questions that are crucial to a fuller understanding of human attention and behavior. We believe that many interesting and yet unexplored questions about attention arise when attention is considered from the perspective of the real world. For example, what is the function of attention in everyday situations? How do people learn to orient their attention to common directional stimuli (e.g., arrows)? What role does attention play in social interactions? How does attention differ among individuals and cultures? These are just a few of the many interesting questions that have been, up to this point, largely ignored.

With these issues in mind, our conclusion is that attention researchers must strike out in a new direction-one that involves studying how attention operates when people are embedded in real-world situations. To get a more complete understanding of the brain and cognitive processes involved in attention, one must move away from studying attention in impoverished settings (e.g., mere flashes of light on a computer screen). Instead, when studying attention, one must consider both (a) the natural characteristics of observers, such as their emotions, goals, and evolutionary histories, and (b) the characteristics of observers' natural everyday environment. Because observers are influenced by the environment, and also influence their experience of the environment, one cannot separate observers from their natural environments when studying attention.

Thus, an important step in this new direction is to reinstate the link between the observer and the natural environment. One way to achieve this is to bring the world back into the laboratory by creating conditions that better approximate situations that people encounter in the real world. Our studies of how eye gaze influences attention provide an example of our first strides in this direction. Note, however, we are not simply suggesting that researchers use everyday stimuli in the same old paradigms. In fact, it is our position that the paradigms themselves do not adequately capture important aspects of natural settings. We believe that ultimately new methods and procedures will have to be developed in order to study attention as it is used in real life.

Another way to restore the link between the observer and the natural environment is to get out of the laboratory and study how individuals actually behave in the real world. This involves observing and describing how attention operates in the real world and using these observations and descriptions to guide new ways of conceptualizing attention. Observing and describing cognition and behavior in the real world has already proven to be fruitful in other areas of study. A wonderful recent example is provided by Güntürkün (2003). He observed kissing couples in public places and recorded that twice as many adults turn their heads to the right as the left when kissing. This lovely result suggests that a rightward head-turning bias, which is present shortly before and after birth, persists into adulthood. This study highlights what Neisser (1982) wrote more than 20 years ago when he argued that psychologists should ground their research in everyday behavior: "We are finding out what really happens in the world around us, and that will be worth knowing in any imaginable future" (p. 10).

\footnotetext{
Recommended Reading

Clark, A. (1997). Being there: Putting brain, body, and world together again. Cambridge, MA: MIT Press.
} 
Neisser, U., \& Hyman, I.E., Jr. (1982). Memory observed. New York: Worth.

Rosch, E. (1999). Reclaiming concepts. Journal of Consciousness Studies, 6, 61-77.

Wilson, M. (2002). Six views of embodied cognition. Psychonomic Bulletin \& Review, 9, 625-636.

\section{Note}

1. Address correspondence to Alan Kingstone, 2136 West Mall, University of British Columbia, Vancouver, Canada V6T1Z4; e-mail: alan.kingstone@ ubc.ca.

\section{References}

Baron-Cohen, S. (1995). Mindblindness: An essay on autism and theory of mind. Cambridge, MA: MIT Press.

Broadbent, D.E. (1971). Decision and stress. London: Academic Press.

Eastwood, J.D., Smilek, D., \& Merikle, P.M. (2001). Differential attentional guidance, by unattended faces expressing positive and negative emotion. Perception \& Psychophysics, 63, 10041013

Friesen, C.K., \& Kingstone, A. (1998). The eyes have it! Reflexive orienting is triggered by nonpredictive gaze. Psychonomic Bulletin $\mathcal{E}$ Review, 5, 490-495.

Güntürkün, O. (2003). Adult persistence of headturning asymmetry. Nature, 421, 711.

Jonides, J. (1981). Voluntary versus automatic control over the mind's eye's movement. In J.B. Long \& A.D. Baddeley (Eds.), Attention and performance IX (pp. 187-203). Hillsdale, NJ: Erlbaum.

Kobayashi, H., \& Kohshima, S. (1997). Unique morphology of the human eye. Nature, 387 767-768

Neisser, U. (1982). Memory: What are the important questions? In U. Neisser \& I.E. Hyman, Jr. (Eds.), Memory observed (pp. 3-18). New York: Worth.

Posner, M.I. (1978). Chronometric explorations of mind. Hillsdale, NJ: Erlbaum.

Ristic, J., Friesen, C.K., \& Kingstone, A. (2002). Are eyes special? It depends on how you look at it. Psychonomic Bulletin \& Review, 9, 507-513.

Ristic, J., Olk, B., Ho, S., \& Kingstone, A. (2003) Endogenous orienting: What have we been measuring? Cognitive Neuroscience Society $A b$ stracts, 10, 55 .

Treisman, A., \& Gelade, G. (1980). A feature integration theory of attention. Cognitive Psychology, 12, 97-136.

\section{Inattentional Blindness: Looking Without Seeing}

\section{Arien Mack ${ }^{1}$}

Psychology Department, New School University, New York, New York

\section{Keywords}

inattention; perception; awareness search shows that we rarely see what we are looking at unless our attention is directed to it. This phenomenon can have serious life-and-death consequences. Although the inextricable link between perceiving and attending was noted long ago by Aristotle, this phenomenon, now called inattentional blindness (IB), only recently has been named and carefully studied. Among the many questions that have been raised about IB are questions about the fate of the clearly visible, yet unseen stimuli, whether any stimuli reliably capture attention, and, if so, what they have in common. Finally, is IB an instance of rapid forgetting, or is it a failure to perceive? second airplane until it was too late to avoid a collision.

As it turns out, such events are not uncommon and even may account for many car accidents resulting from distraction and inattention. This is why talking on cell telephones while driving is a distinctly bad idea. However, the pervasive assumption that the eye functions like a camera and our subjective impression of a coherent and richly detailed world lead most of us to assume that we see what there is to be seen by merely opening our eyes and looking. Perhaps this is why we are so astonished by events like the airplane scenario, although less potentially damaging instances occur every day, such as when we pass by a friend without seeing her.

These scenarios are examples of what psychologists call inattentional blindness (IB; Mack \& Rock, 1998). IB denotes the failure to see highly visible objects we may be looking at directly when our attention is elsewhere. Although IB is a visual phenomenon, it has auditory and tactile counterparts as well; for example, we often do not hear something said to us if we are "not listening." 


\section{INATTENTIONAL BLINDNESS}

The idea that we miss a substantial amount of the visual world at any given time is startling even though evidence for such selective seeing was first reported in the 1970s by Neisser (1979). In one of several experiments, he asked participants to view a video of two superimposed ball-passing games in which one group of players wore white uniforms and another group wore black uniforms. Participants counted the number of passes between members of one of the groups. When the participants were subsequently asked to report what they had seen, only $21 \%$ reported the presence of a woman who had unexpectedly strolled though the basketball court carrying an open umbrella, even though she was clearly in view some of the time. Researchers recently replicated this finding with a study in which a man dressed in a gorilla costume stopped to thump his chest while walking through the court and remained visible for between 5 and 9 s (Simons \& Chabris, 1999).

Although it is possible that some failures to see the gorilla or the umbrella-carrying woman might have resulted from not looking directly at them, another body of work supports the alternative explanation that the observers were so intent on counting ball passes that they missed the unexpected object that appeared in plain view. Research I have conducted with my colleagues (Mack \& Rock, 1998) conclusively demonstrates that, with rare exceptions, observers generally do not see what they are looking directly at when they are attending to something else. In many of these experiments, observers fixated on specified locations while simultaneously attending to a demanding perceptual task, the object of which might be elsewhere. Under these conditions, observers often failed to perceive a clearly visible stimulus that was located exactly where they were looking.

\section{INATTENTIONAL BLINDNESS OR INATTENTIONAL AMNESIA?}

Not surprisingly, there is a controversy over whether the types of failures documented in such experiments are really evidence that the observers did not see the stimulus, or whether they in fact saw the stimulus but then quickly forgot it. In other words, is IB more correctly described as inattentional amnesia (Wolfe, 1999)? Although this controversy may not lend itself to an empirical resolution, many researchers find it difficult to believe that a thumping gorilla appearing in the midst of a ball game is noticed and then immediately forgotten. What makes the argument for inattentional amnesia even more difficult to sustain is evidence that unseen stimuli are capable of priming, that is, of affecting some subsequent act. (For example, if a subject is shown some object too quickly to identify it and is then shown it again so that it is clearly visible, the subject is likely to identify it more quickly than if it had not been previously flashed. This is evidence of priming: The first exposure speeded the response to the second.) Priming can occur only if there is some memory of the stimulus, even if that memory is inaccessible.

\section{UNCONSCIOUS PERCEPTION}

A considerable amount of research has investigated unconscious, or implicit, perception and those perceptual processes that occur outside of awareness. This work has led many researchers to conclude that events in the envi- ronment, even if not consciously perceived, may direct later behavior. If stimuli not seen because of IB are in fact processed but encoded outside of awareness, then it should be possible to demonstrate that they prime subsequent behavior.

The typical method for documenting implicit perception entails measuring reaction time over multiple trials. Such studies are based on the assumption that an implicitly perceived stimulus will either speed up or retard subsequent responses to relevant stimuli depending on whether the priming produces facilitation or inhibition. ${ }^{2}$ However, because subjects in IB experiments cannot be made aware of the critical stimulus, unlike in many kinds of priming studies, only one trial with that stimulus is possible. This requirement rules out reaction time procedures, which demand hundreds of trials because reaction time differences tend to be small and therefore require stable response rates that can be achieved only with many trials. Fortunately, an alternate procedure, stem completion, can be used when the critical stimuli are words. In this method, some observers (experimental group) are exposed to a word in an IB procedure, and other observers (control group) are not. Then, the initial few letters of the unseen word are presented to all the observers, who are asked to complete the string of letters with one or two English words. If the members of the experimental group complete the string with the unseen word more frequently than do the members of the control group, this is taken as evidence that the experimental group implicitly perceived and encoded the word.

IB experiments using this method have demonstrated significant priming (Mack \& Rock, 1998), as well as other kinds of evidence that visual information undergoes substantial processing prior to the engagement of attention. For exam- 
ple, evidence that aspects of visual processing take place before attention is allocated has been provided by a series of ingenious IB experiments by Moore and her collaborators (e.g., Moore \& Egeth, 1997). This work has shown that under conditions of inattention, basic perceptual processes, such as those responsible for the grouping of elements in the visual field into objects, are carried out and influence task responses even though observers are unable to report seeing the percepts that result from those processes. For example, in one study using a modification of the IB procedure, Moore and Egeth investigated the Müller-Lyer illusion, in which two lines of equal length look unequal because one has outgoing fins, which make it look longer, and the other has ingoing fins, which make it look shorter. In this case, the fins were formed by the grouping of background dots: Dots forming the fins were closer together than the other dots in the background. Moore and Egeth demonstrated that subjects saw the illusion even when, because of inattention, the fins were not consciously perceived. Whatever processes priming entails, the fact that it occurs is evidence of implicit perception and the encoding of a stimulus in memory. Thus, the fact that the critical stimulus in the IB paradigm can prime subsequent responses is evidence that this stimulus is implicitly perceived and encoded.

\section{When Do Stimuli Capture Attention and Why?}

That unconsciously perceived stimuli in IB experiments undergo substantial processing in the brain is also supported by evidence that the select few stimuli able to capture attention when attention is elsewhere are complex and meaningful (e.g., the observer's name, an iconic image of a happy face) rather than simple features like color or motion. This fact suggests that attention is captured only after the meaning of a stimulus has been analyzed. There are psychologists who believe that attention operates much earlier in the processing of sensory input, before meaning has been analyzed (e.g., Treisman, 1969). These accounts, however, do not easily explain why modest changes, such as inverting a happy face and changing one internal letter in the observer's name, which alter the apparent meaning of the stimuli but not their overall shape, cause a very large increase in IB (Mack \& Rock, 1998).

\section{Meaning and the Capture of Attention}

If meaning is what captures attention, then it follows axiomatically that meaning must be analyzed before attention is captured, which is thought to occur at the end stage of the processing of sensory input. This therefore implies that even those stimuli that we are not intending to see and that do not capture our attention must be fully processed by the brain, for otherwise their meanings would be lost before they had a chance of capturing our attention and being perceived. If this is the case, then we are left with some yet-unanswered, very difficult questions. Are all the innumerable stimuli imaged on our retinas really processed for meaning and encoded into memory, and if not, which stimuli are and which are not?

Although we do not yet have answers to these questions, an unpublished doctoral dissertation by Silverman, at New School University, has demonstrated that there can be priming by more than one element in a multielement display, even when these elements cannot be reported by the subject. This finding is relevant to the question of whether all elements in the visual field are processed and stored because up to now there has been scarcely any evidence of priming by more than one unreportable element in the field. The fact of multielement priming begins to suggest that unattended or unseen elements are processed and stored, although it says nothing about how many elements are processed and whether the meaning of all the elements is analyzed.

One answer to the question of how much of what is not seen is encoded into memory comes from an account of perceptual processing based on the assumption that perception is a limited-capacity process and that processing is mandatory up to the point that this capacity is exhausted (Lavie, 1995). According to this analysis, the extent to which unattended objects are processed is a function of the difficulty of the perceptual task (i.e., the perceptual load). When the perceptual load is high, only attended stimuli are encoded. When it is low, unattended stimuli are also processed. This account faces some difficulty because it is not clear how perceptual load should be estimated. Beyond this, however, it is difficult to reconcile this account with evidence suggesting that observers are likely to see their own names even when they occur among the stimuli that must be ignored in order to perform a demanding perceptual task (Mack, Pappas, Silverman, \& Gay, 2002). It should be noted, however, that these latter results are at odds with a published report (Rees, Russell, Firth, \& Driver, 1999) I describe in the next section.

\section{EVIDENCE FROM NEURAL IMAGING}

Researchers have used magnetic imaging techniques to try to 
determine what happens in the brain when observers fail to detect a visual stimulus because their attention is elsewhere. Neural recording techniques may be able to show whether visual stimuli that are unconsciously perceived arouse the same areas of the brain to the same extent as visual stimuli that are seen. This is an important question because it bears directly on the nature of the processing that occurs outside of awareness prior to the engagement of attention and on the difference between the processing of attended and unattended stimuli.

In one study, Scholte, Spekreijse, and Lamme (2001) found similar neural activity related to the segregation of unattended target stimuli from their backgrounds (i.e., the grouping of the unattended stimuli so they stood out from the background on which they appeared), an operation that is thought to occur early in the processing of visual input. This activation was found regardless of whether the stimuli were attended and seen or unattended and not seen, although there was increased activation for targets that were attended and seen. This finding is consistent with the behavioral findings of Moore and Egeth (1997), cited earlier, showing that unattended, unseen stimuli undergo lower-level processing such as grouping, although the additional neural activity associated with awareness suggests that there may be important differences in processing of attended versus unattended stimuli.

In another study, Rees and his colleagues (Rees et al., 1999) used functional magnetic resonance imaging (fMRI) to picture brain activity while observers were engaged in a perceptual task. They found no evidence of any difference between the neural processing of meaningful and meaningless lexical stimuli when they were ignored, although when the same stimuli were at- tended to and seen, the neural processing of meaningful and meaningless stimuli did differ. These results suggest that unattended stimuli are not processed for meaning. However, in another study that repeated the procedure used by Rees et al. (without fMRI recordings) but included the subject's own name among the ignored stimuli, many subjects saw their names, suggesting that meaning was in fact analyzed (Mack et al., 2002). Thus, one study shows that ignored stimuli are not semantically processed, and the other suggests that they are. This conflict remains unresolved. Are unattended, unseen words deeply processed outside of awareness, despite these fMRI results, which show no evidence of semantic neural activation by ignored words? How can one reconcile behavioral evidence of priming by lexical stimuli under conditions of inattention (Mack \& Rock, 1998) with evidence that these stimuli are not semantically processed?

\section{NEUROLOGICAL DISORDER RELATED TO INATTENTIONAL BLINDNESS}

People who have experienced brain injuries that cause lesions in the parietal cortex (an area of the brain associated with attention) often exhibit what is called unilateral visual neglect, meaning that they fail to see objects located in the visual field opposite the site of the lesion. That is, for example, if the lesion is on the right, they fail to eat food on the left side of their plates or to shave the left half of their faces. Because these lesions do not cause any sensory deficits, the apparent blindness cannot be attributed to sensory causes and has been explained in terms of the role of the parietal cortex in attentional processing (Rafal, 1998). Visual neglect therefore seems to share important similarities with IB. Both phenomena are attributed to inattention, and there is evidence that in both visual neglect (Rafal, 1998) and IB, unseen stimuli are capable of priming. In IB and visual neglect, the failure to see objects shares a common cause, namely inattention, even though in one case the inattention is produced by brain damage, and in the other the inattention is produced by the task. Thus, evidence of priming by neglected stimuli appears to be additional evidence of the processing and encoding of unattended stimuli.

\section{ATTENTION AND PERCEPTION}

IB highlights the intimate link between perception and attention, which is further underscored by recent evidence showing that unattended stimuli that share features with task-relevant stimuli are less likely to suffer IB than those that do not (Most et al., 2001). This new evidence illustrates the power of our intentions in determining what we see and what we do not.

\section{CONCLUDING REMARKS}

Although the phenomenon of IB is now well established, it remains surrounded by many unanswered questions. In addition to the almost completely unexplored question concerning whether all unattended, unseen stimuli in a complex scene are fully processed outside of awareness (and if not, which are and which are not), there is the question of whether the observer can locate where in the visual field the information extracted 
from a single unseen stimulus came from, despite the fact that the observer has failed to perceive it. This possibility is suggested by the proposal that there are two separate visual systems, one dedicated to action, which does not entail consciousness, and the other dedicated to perception, which does entail consciousness (Milner \& Goodale, 1995). That is, the action stream may process an unseen stimulus, including its location information, although the perception stream does not. An answer to this question would be informative about the fate of the unseen stimuli.

The pervasiveness of IB raises another unresolved question. Given that people see much less than they think they do, is the visual world a mere illusion? According to one provocative answer to this question, most recently defended by O'Regan and Noe (2001), the outcome of perceptual processing is not the construction of some internal representation; rather, seeing is a way of exploring the environment, and the outside world serves as its own external representation, eliminating the need for internal representations. Whether or not this account turns out to be viable, the phenomenon of IB has raised a host of questions, the answers to which promise to change scientists' understanding of the nature of perception. The phenomenon itself points to the serious dangers of inattention.

\section{Recommended Reading}

Mack, A., \& Rock, I. (1998). (See References)

Rensink, R. (2002). Change blindness. Annual Review of Psychology, 53, 245-277.

Simons, D. (2000). Current approaches to change blindness. $V i$ sual Cognition, 7, 1-15.

Wilkens, P. (Ed.). (2000). Symposium on Mack and Rock's Inattentional Blindness. Psyche, 6 and 7. Retrieved from http://psyche.cs. monash.edu.au/psyche-indexv7.html\#ib

Acknowledgments-I am grateful for the comments and suggestions of Bill Prinzmetal and Michael Silverman.

\section{Notes}

1. Address correspondence to Arien Mack, Psychology Department, New School University, 65 Fifth Ave., New York, NY 10003.

2. An example of a speeded-up response (facilitation, or positive priming) has already been given. Negative, or inhibition, priming occurs when a stimulus that has been actively ignored is subsequently presented. For example, if a series of superimposed red and green shapes is rapidly presented and subjects are asked to report a feature of the red shapes, later on it is likely to take them longer to identify the green shapes than a shape that has not previously appeared, suggesting that the mental representation of the green shapes has been associated with something like an "ignore me" tag.

\section{References}

Haines, R.F. (1991). A breakdown in simultaneous information processing. In G. Obrecht \& L.W. Stark (Eds.), Presbyopia research (pp. 171-175). New York: Plenum Press.

Lavie, N. (1995). Perceptual load as a necessary condition for selective attention. Journal of Experimental Psychology: Human Perception and Performance, 21, 451-468.

Mack, A., Pappas, Z., Silverman, M., \& Gay, R. (2002). What we see: Inattention and the capture of attention by meaning. Consciousness and Cognition, 11, 488-506.

Mack, A., \& Rock, I. (1998). Inattentional blindness. Cambridge, MA: MIT Press.

Milner, D., \& Goodale, M.A. (1995). The visual brain in action. Oxford, England: Oxford University Press.

Moore, C.M., \& Egeth, H. (1997). Perception without attention: Evidence of grouping under conditions of inattention. Journal of Experimental Psychology: Human Perception and Performance, 23, 339-352.

Most, S.B., Simons, D.J., Scholl, B.J., Jimenez, R. Clifford, E., \& Chabris, C.F. (2001). How not to be seen: The contribution of similarity and selective ignoring to sustained inattentional blindness. Psychological Science, 12, 9-17.

Neisser, U. (1979). The control of information pickup in selective looking. In A.D. Pick (Ed.), Perception and its development: A tribute to Eleanor Gibson (pp. 201-219). Hillsdale, NJ: Erlbaum.

O'Regan, K., \& Noe, A. (2001). A sensorimotor account of vision and visual consciousness. Behavioral and Brain Sciences, 25, 5.

Rafal, R. (1998). Neglect. In R. Parasuraman (Ed.), The attentive brain (pp. 489-526). Cambridge, MA: MIT Press.

Rees, G., Russell, C., Firth, C., \& Driver, J. (1999) Inattentional blindness versus inattentional amnesia. Science, 286, 849-860.

Scholte, H.S., Spekreijse, H., \& Lamme, V.A. (2001). Neural correlates of global scene segmentation are present during inattentional blindness [Abstract]. Journal of Vision, 1(3), Article 346. Retrieved from http://journalofvision. org $/ 1 / 3 / 346$

Simons, D.J., \& Chabris, C.F. (1999). Gorillas in our midst: Sustained inattentional blindness for dynamic events. Perception, 28, 1059-1074.

Treisman, A. (1969). Strategies and models of selective attention. Psychological Review, 76, 282-299.

Wolfe, J. (1999). Inattentional amnesia. In V. Coltheart (Ed.), Fleeting memories (pp. 71-94). Cambridge, MA: MIT Press. 